\title{
Targeted silencing of the Aquaporin 2 gene of Rhipicephalus (Boophilus) microplus reduces tick fitness
}

Hala E. Hussein ${ }^{1,2}$, Glen A. Scoles ${ }^{3}$, Massaro W. Ueti ${ }^{3}$, Carlos E. Suarez ${ }^{3}$ Fatma K. Adham², Felix D. Guerrero 4,5 and Reginaldo G. Bastos ${ }^{6^{*}}$

\begin{abstract}
Background: Ticks are blood-feeding arthropods that can affect human and animal health both directly by blood-feeding and indirectly by transmitting pathogens. The cattle tick Rhipicephalus (Boophilus) microplus is one of the most economically important ectoparasites of bovines worldwide and it is responsible for the transmission of the protozoan Babesia bovis, the etiological agent of bovine babesiosis. Aquaporins (AQPs) are water channel proteins implicated in physiological mechanisms of osmoregulation. Members of the AQP family are critical for blood-feeding arthropods considering the extreme osmoregulatory changes that occur during their feeding. We investigated the pattern of expression of a newly identified AQP2 gene of $R$. microplus (RmAQP2) in different tick tissues and stages. We also examined in vivo the biological implications of silencing expression of RmAQP2 silencing during tick feeding on either uninfected or B. bovis-infected cattle.
\end{abstract}

Methods: In silico gene analyses were performed by multiple alignments of amino acid sequences and topology prediction. Levels of RmAQP2 transcripts in different tick tissues and stages were analyzed by reverse transcriptase quantitative PCR. Patterns of expression of RmAQP2 protein were investigated by immunoblots. Gene silencing was performed by RNA interference and in vivo functional analyses carried out by feeding ticks on either uninfected or B. bovis-infected cattle.

Results: RmAQP2 transcripts were found in unfed larvae, engorged nymphs, and salivary glands and guts of partially engorged females; however, of all tick tissues and stages examined, RmAQP2 protein was found only in salivary glands of partially engorged females. RMAQP2 silencing significantly reduced tick fitness and completely abrogated protein expression. The effect of RMAQP2 silencing on fitness was more pronounced in females fed on a B. bovis-infected calf than in ticks fed on an uninfected calf and none of their larval progeny survived.

Conclusions: Collectively, considering the gene expression and tick fitness data, we conclude that RmAQP2 is critical for tick blood feeding and may be a suitable candidate target for the development of novel strategies to control $R$. microplus and tick-borne parasites.

Keywords: Rhipicephalus (Boophilus) microplus, Aquaporin, Tick fitness, RNA interference, Gene silencing, Babesia bovis

\footnotetext{
* Correspondence: rgbastos@vetmed.wsu.edu

${ }^{6}$ School of Molecular Biosciences, Washington State University, Pullman, WA 99164, USA

Full list of author information is available at the end of the article
}

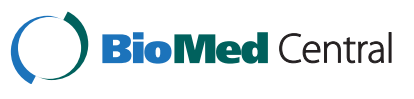

(c) 2015 Hussein et al. Open Access This article is distributed under the terms of the Creative Commons Attribution 4.0 International License (http://creativecommons.org/licenses/by/4.0/), which permits unrestricted use, distribution, and reproduction in any medium, provided you give appropriate credit to the original author(s) and the source, provide a link to the Creative Commons license, and indicate if changes were made. The Creative Commons Public Domain Dedication waiver (http://creativecommons.org/publicdomain/zero/1.0/) applies to the data made available in this article, unless otherwise stated. 


\section{Background}

Aquaporins (AQPs) are a family of transmembrane proteins that form pores to transport water and small solutes across cellular membranes [1]. Members of the AQP family have been identified throughout the plant and animal kingdoms [2]. The structures of AQPs are highly conserved among species, consisting of six transmembrane domains that are connected by two intracellular loops and three extracellular loops. Two asparagine-proline-alanine (NPA) motifs are considered AQP signature motifs and are located at the protein portion that interacts to form a pore [1]. A total of $13 \mathrm{AQP}$ members have been identified so far and classed into two subsets: those that are permeated by water and those that are permeated by water plus other small molecules, such as glycerol and urea $[1,2]$.

Numerous members of the AQP family have been identified in arthropods in the last few years due to the availability of several arthropod genomes and genetic information, such as transcriptomes and cDNA libraries [3]. Studies have shown that AQPs play a pivotal role in arthropods, especially in blood-feeding species, such as mosquitoes and ticks. In fact, blood-feeding arthropods have become a model for AQP research due to the critical relevance that osmoregulation plays during feeding [4]. Tick females can take on up to 100 times their body weight in blood, and concentrate the blood meal by returning approximately $75 \%$ of the ingested water and ions via their saliva into the host [5]. Therefore, the osmoregulatory system of ticks is central for their parasitic life cycle and has implications for efficient feeding and subsequent production of viable offspring.

The cattle tick Rhipicephalus (Boophilus) microplus is one of the most economically important ectoparasites of bovines, as it is the main vector of the apicomplexan protozoan Babesia bovis, the etiological agent of bovine babesiosis [6]. Adult females $R$. microplus acquire $B$. bovis merozoites by ingesting blood from an infected bovine and pass the protozoan transovarially to their larval progeny, which then can transmit B. bovis sporozoites to naïve cattle during subsequent feeding [6-8]. In most endemic areas control of bovine babesiosis transmission relies mainly on targeting populations of $R$. microplus with acaricide treatments and to a lesser extent with the use of live attenuated vaccines [6-10]. However, the efficacy of commercial anti-tick vaccines is inconsistent in different regions of the world and the recent development of tick populations resistant to acaricides represents a serious threat to the cattle industry [11, 12]. Additionally, the re-emergence of $R$. microplus in areas that had been considered to be free of this tick, such as the regions outside the permanent quarantine zone in south Texas, USA, is causing concerns about the reestablishment of active $B$. bovis transmission in areas currently free of bovine babesiosis. Exposure of naïve cattle in these areas to B. bovis would lead to significant mortality since no herd immunity is present in the population.

Intake of blood meals leading to full engorgement of adult females and subsequent production of viable larval offspring are critical steps in the tick life cycle. A better understanding of mechanisms involved in these processes may lead to the identification of novel targets to control ticks and tick-borne parasites. In the present study we investigated the pattern of expression of a newly identified $A Q P 2$ gene of $R$. microplus (RmAQP2). Our findings showed that $R m A Q P 2$ is transcribed in unfed larvae, engorged nymphs, and salivary glands and guts of partially engorged females. However, among the tick tissues and stages analyzed, RmAQP2 protein was only found in salivary glands of partially engorged females. In vivo analyses demonstrated that silencing of RmAQP2 with RNA interference significantly reduced tick fitness and this effect was even more dramatic in females fed on a calf during acute B. bovis infection than in ticks fed on an uninfected calf.

\section{Methods}

\section{Cattle, ticks and protozoan}

Holstein calves 3-4 months of age, tested negative for $B$. bovis by PCR [13] and cELISA [14], were used in this study. The animals were maintained according to protocols approved by the University of Idaho Institutional Animal Care and Use Committee. Ticks from our laboratory colony, Rhipicephalus microplus La Minita strain [15] were tested by sequencing the cytochrome oxidase 1 (Cox 1$)$ gene to confirm that they were of the same strain as those used to obtain the sequences from the $R$. microplus gene index project $[16,17]$. For tick strain identification, genomic DNA was prepared from R. microplus male ticks. PCR using the Cox 1 external and nested primers (Table 1) and prepared with FastStart Taq reagents (Roche Diagnostics, Indianapolis, IN) was carried out under the following conditions for both rounds: $95{ }^{\circ} \mathrm{C}$ for $5 \mathrm{~min}$; 35 cycles of $95{ }^{\circ} \mathrm{C}$ for $1 \mathrm{~min}$, $65{ }^{\circ} \mathrm{C}$ for $1 \mathrm{~min}$, and $72{ }^{\circ} \mathrm{C}$ for $2 \mathrm{~min}$; final extension at $72{ }^{\circ} \mathrm{C}$ for $5 \mathrm{~min}$. The nested PCR product was cloned into a $\mathrm{pCR}^{\mathrm{m}} 4 \mathrm{TOPO}^{\circ}$ plasmid for sequencing (Invitrogen, Carlsbad, CA). The Cox1 sequence obtained from our tick colony had $100 \%$ identity with the mitochondrial genome sequence of the $R$. microplus Deutsch strain from Texas, USA [GenBank: KP143546], which was used to obtain the RmAQP2 sequence, and confirming that our laboratory colony represents a prototypical strain of $R$. microplus [18].

To obtain unfed adult ticks for each experiment, approximately 40,000 larvae from $2 \mathrm{~g}$ of eggs were placed under a cloth patch on uninfected calves. On day 13-14, engorged nymphs were manually removed and held in 
Table 1 Primer purposes and sequences, gene region and PCR product size for Rhipicephalus microplus Cox1 and RmAQP2

\begin{tabular}{lllll}
\hline Primer purpose & Forward primer & Reverse primer & Gene region & Product size (bp) \\
\hline Cox1 amplification external & atttaccgcgatgaatatactc & gcaaggcctaaaaaatg & 1 to 1298 & 1298 \\
Cox1 amplification nested primers & ctcaactaatcataaagacattgg & gctaatataattcctgttaaacctcc & 21 to 1072 & 1052 \\
RMAQP2 dsRNA synthesis & aattcagcagcaggagaagc & cggcgtacaccaggtaaact & 17 to 414 & 397 \\
RmAQP2 dsRNA synthesis & cctctcctcgtcggcctca & cggctaaaacgcaaaaaggt & 614 to 1009 & 396 \\
RMAQP2 Real-time PCR & gtaagtcaccgcacagta & tacacaatagcgaggtt & 349 to 453 & 105 \\
\hline
\end{tabular}

an incubator at $25{ }^{\circ} \mathrm{C}$ with $96 \% \mathrm{RH}$ to molt to adults. After 2-3 days of incubation, freshly molted unfed adult females and males were sorted out and used for evaluation of gene expression. The first silencing experiment was carried out by placing injected ticks on a calf infected with Babesia bovis. The second silencing experiment was done on an uninfected calf. For the experiment with the Babesia bovis infected animal, the calf was injected with a stabilate containing approximately $1.4 \times 10^{8} \mathrm{~B}$. bovisinfected erythrocytes (T2Bo strain) [19]. The infected calf was monitored daily for the presence of $B$. bovis in peripheral blood and clinical signs of babesiosis. Parasitemia of B. bovis in peripheral blood was examined by qPCR to amplify the single copy msa- 1 gene as previously described [13]. The B. bovis-infected animal presented clinical indications of acute $B$. bovis infection including a drop in PCV, fever and detection of parasites in peripheral blood by qPCR (data not shown).

\section{In silico analysis}

Nucleotide sequence encoding for RmAQP2 cDNA was obtained from the $R$. microplus gene index project $[16,17]$ [GenBank ID: KP406519]. Multiple alignments of amino acid sequences were generated using the Multiple Alignment Module of LaserGene of DNASTAR software ${ }^{-}$ (DNASTAR, Inc.). The Simple Modular Architecture Research Tool (SMART) [20] and the Transmembrane Hidden Markov Model package 2 (TMHMM2) [21] were used to predict domains and signal peptides in the RmAQP2 protein sequences. The tools Transmembrane Protein Representation in 2 Dimensions (TMRPres2) [22] and Swiss-model [23] were used to create 2D and 3D (respectively) visual representations of the RmAQP2 protein for topology analyses.

\section{Reverse transcriptase real-time PCR}

A reverse transcriptase quantitative real-time PCR (RTqPCR) was standardized to assess the level of expression of $R m A Q P 2$ in different tick tissues and stages. In order to make solid significant observations regarding gene expression, $\mathrm{qPCR}$ was designed using the pertinent requirements of the minimum information for publication of qPCR experiments [24]. Unfed larvae, engorged nymphs, unfed male ticks, and salivary glands, guts and ovaries from partially engorged tick females were collected in RNAlater
(Ambion) and stored at $-20{ }^{\circ} \mathrm{C}$ following the manufacturer's protocol. Total RNA was extracted using the RNAqueous $^{\circ}$ Kit (Ambion) according to the manufacturer's protocol and quantified by Qubit ${ }^{\mathrm{tm}}$ fluorometer. Two hundred nanograms of total RNA were utilized for cDNA synthesis using the Superscript ${ }^{\circ}$ Vilo $^{\text {Tn }}$ cDNA Synthesis Kit (Invitrogen) following the manufacturer's protocol. Technical replicates were performed to evaluate enzymatic variations during the synthesis of cDNA in a given RNA sample. The RmAQP2 cDNA sequence was used to design qPCR primers to amplify a fragment of 105 base pairs (Table 1). Quantitative real-time PCR were performed in a CFX96 $^{\text {ti }}$ Real-Time PCR Detection System using the SsoFast $^{\mathrm{Tm}}$ EvaGreen $^{\circ}$ Supermix (Bio-Rad). Cycling conditions consisted of an enzyme activation step of $95^{\circ} \mathrm{C}$ for $30 \mathrm{~s}$ followed by 40 cycles of denaturation at $95^{\circ} \mathrm{C}$ for $5 \mathrm{~s}$ and annealing/extension of $60{ }^{\circ} \mathrm{C}$ for $5 \mathrm{~s}$. Reactions were performed in duplicate in $20 \mu \mathrm{l}$ using $200 \mathrm{nM}$ of each primer and $2 \mu \mathrm{l}$ of a $1 / 20$ dilution of cDNA as template. The CFX Manager ${ }^{\mathrm{Tm}}$ Software (Bio-Rad) was used to analyze the RT-qPCR data. Gene expression was normalized to the total amount of RNA used to generate the cDNA, as xpreviously described [13]. The RmAQP2 transcription level was then calculated as a relative expression using the formula: Relative expression (sample) $=2^{[C q \text { (control) }-C q \text { (sample) }]}$, where the control is either the highest or lowest $\mathrm{C} q$ value for the gene of interest, as previously described [13, 25]. Melt curve analyses showed the absence of primer dimers and nonspecific amplification. The absence of PCR product in no RNA control reactions and in no transcriptase reverse control reactions indicated the specificity of the $R m A Q P 2$ RT-qPCR (data not shown).

\section{Monoclonal antibody production and immunobloting assays}

Three synthetic peptides from the extracellular loops of RmAQP2 were manufactured by BioSynthesis, Inc. (Texas, USA) (Additional file 1: Figure S1). Peptide 1: LGSVGLAAAP (10-mer) (Amino acids 52 to 61). Peptide 2: ADALSQVDVNLAIVYGTNATAPVFSCF PAPGV (32-mer) (Amino acids 125 to 156). Peptide 3: MCGWGSAVFSFRSYNWFWV (19-mer) (Amino acids 229 to 247). Three 6-week-old BALB/c female mice were immunized subcutaneously with $50 \mathrm{mg}$ of a pool of all three RmAQP2 peptides diluted in $0.1 \mathrm{ml}$ of sterile PBS 
emulsified with an equal volume of TiterMax ${ }^{\circ}$ Gold Adjuvant (Sigma-Aldrich). The primary immune response was boosted twice by subcutaneous immunization at 15-day intervals with the same concentration of peptide antigen plus adjuvant. Mice immune responses were monitored by ELISA using the RmAQP2 peptides as antigens. Three days prior to cell fusion, mice were boosted intravenously with the same concentration of antigen without adjuvant. Cell fusions and cloning by limiting dilution were performed by standard procedures [26]. Supernatants from the initial fusion and from clones obtained by limiting dilution were screened by ELISA. A total of 10 hybridoma clones were obtained and the hybridoma supernatants used to assess the expression of RmAQP2 in tick tissues and stages by immunoblot. For the immunoblot, antigens were prepared from unfed larvae, engorged nymphs, and salivary glands and guts of partially engorged females (at 5 days of feeding). Tick tissues and stages were homogenized in lysis buffer-Nonidet-P40 (NP-40) $(150 \mathrm{mM}$ sodium chloride $1.0 \%$ NP-40 - $50 \mathrm{mM}$ Tris, $\mathrm{pH} 8.0)$ and protease inhibitor $(1 \mu \mathrm{g} / \mathrm{ml})$. Total protein was quantified by Micro BCA Protein Assay (Thermo Scientific Pierce) and $3 \mu \mathrm{g}$ of total protein were separated on 4-20\% Mini-PROTEAN ${ }^{\ominus}$ TGX $^{\mathrm{Tm}}$ Precast Gels (Bio-Rad) under reducing conditions. Proteins were transferred to a nitrocellulose membrane (Whatman, Dassel, Germany) for $1 \mathrm{~h}$ at $100 \mathrm{~V}$. The membrane was blocked with $5 \%$ skim milk in TBS (Tris-buffered saline: $25 \mathrm{mmol} \mathrm{l}^{-1}$ Tris- $\left.\mathrm{HCl}, 150 \mathrm{mmol} \mathrm{l}^{-1} \mathrm{NaCl}, \mathrm{pH} 7.6\right)$ for $1 \mathrm{~h}$ at room temperature, washed three times in TBS and incubated $1 \mathrm{~h}$ with the appropriate hybridoma supernatant as the primary antibody. The membrane was washed three times with TBS and incubated 30 min with anti-mouse HRP conjugated secondary antibody, then washed again three times with TBS and developed using chemiluminescent HRP antibody detection reagents.

\section{Synthesis of double stranded RNA}

Synthesis of double stranded RNA (dsRNA) was performed as previously described [13]. Specific primers were designed based on the cDNA sequence of the RmAQP2 gene described in the $R$. microplus Gene Index Project [17] (Table 1). Two fragments of approximately $400 \mathrm{bp}$ were amplified, one located at the $5^{\prime}$ and another one located at the $3^{\prime}$ of the $R m A Q P 2$ gene (Additional file 1: Figure S1). PCR products were cloned into $\mathrm{pCR}^{\mathrm{m}} \mathrm{II}-\mathrm{TOPO}^{\circ}$ (Invitrogen), sequenced and used for in vitro transcription. The MEGAscript ${ }^{\circ}$ Transcription Kit (Ambion) was used for the dsRNA synthesis following the manufacturer's protocol. The two RmAQP2 dsRNA molecules were checked by electrophoresis on agarose gel, quantified by spectrophotometry and kept at $-20{ }^{\circ} \mathrm{C}$ until used for tick injection.

\section{Injection of ticks with double stranded RNA and assessment of gene silencing}

Freshly molted unfed females were used for the dsRNA injection. Two independent experiments were run, each with a control group of 200 ticks injected with buffer alone and an experimental group with 200 female ticks each injected with either one or two dsRNA segments identical to $R m A Q P 2$ (Additional file 1: Figure S1). For the first experiment, adult female ticks were injected with one segment of dsRNA from the 3' end of the gene and fed on B. bovis-infected calf. For the second experiment, adult female ticks were injected with both segments of dsRNA, one from from the $5^{\prime}$ end and one from the $3^{\prime}$ end of the gene, then fed to repletion on an uninfected calf (B. bovisfree animal). Individual females were injected with $1 \mu \mathrm{l}$ total dsRNA (approximately $1 \times 10^{11}$ molecules dissolved in $0.1 \mathrm{mM}$ EDTA buffer) or buffer control (0.1 mM EDTA buffer) through the coxal membrane at the base of the $4^{\text {th }}$ leg on the right ventral side, as previously described [13]. Ticks injected with the two different dsRNA segments received $0.5 \times 10^{11}$ of each molecule for a total of $1 \times 10^{11}$ molecules. Injections were accomplished using a $10 \mu \mathrm{l}$ syringe with a 33 gauge needle (Hamilton, Bonaduz, Switzerland) and the microprocessor controlled UMP3 injection pump apparatus (World Precision Instruments, Berlin, Germany). After the injection, the dsRNA-injected females, plus an equal number of males, and the bufferinjected females, plus an equal number of males, were placed under separate stockinet sleeves glued to the calf to feed on either a B. bovis-infected calf (experiment 1) or an uninfected calf (experiment 2). For both experiments silencing level of RmAQP2 was investigated using RT-qPCR as described above. Five days after the dsRNA injection, 20 partially engorged female ticks from each group were collected for dissection. Tissue collection, extraction of total RNA, cDNA Synthesis, and qPCR were performed as described above.

\section{Evaluation of tick fitness}

After the dsRNA injection, individual stockinet sleeves were checked daily for the presence of engorged female ticks. Fully engorged females were collected, weighed and put in individual wells in 24-well plates at $26^{\circ} \mathrm{C}$ for oviposition. At day 14 after the beginning of oviposition, egg masses laid by each individual female were weighed and put in individual vials to evaluate hatching. Hatching was evaluated at 30 days after the egg masses were weighed and hatching positive was defined as the presence of any larvae from eggs of an individual female. The larval progeny were maintained in individual vials at $26{ }^{\circ} \mathrm{C}$ for 45 days and the larval survival was determined as the presence of any live larvae among larval progeny from each individual female. 


\section{Statistical analysis}

Weights of engorged females and egg masses were compared by Student's $t$-test (GraphPad Instat', version 3.06, GraphPad Software, Inc., San Diego, CA, USA). The percentages of engorged females, oviposition, hatching and larvae survival were compared by Chi-squared (GraphPad Instat ${ }^{\circ}$ ). Gene expression in different tick tissues and stages was compared by one-way ANOVA and Tukey post-hoc test.

\section{Results}

\section{In silico sequence analysis of the $R$. microplus AQP2}

Multiple alignment analysis revealed that the RmAQP2 amino acid sequence [GenBank ID: KP406519] presents $41.2,86.0,53.0,53.5,54.1,65.5$ percentage identity to $R$. microplus AQP1 [GenBank ID: KJ626366.1], Dermacentor variabilis AQP9 [GenBank ID: ABI53034.1], R. sanguineus AQP1 [GenBank ID: CAR66115.1], Ixodes ricinus AQP1 [GenBank ID: CAX48964.1], I. scapularis AQP1 [GenBank ID: XP_002399532.1] and I. scapularis AQP2 [GenBank ID: XP_002400655.1], respectively (Fig. 1). Considering domain organization, it is important to mention the presence of two AQP signature NPA motifs in the RmAQP2 sequence as well as in all the other AQP's sequences mentioned above, except in the $I$. scapularis AQP2. The two AQP NPA motifs are present in RmAQP2 at the amino acid positions 84 to 86 and 216 to 218 . Topology prediction indicates that RmAQP2 has six transmembrane-spanning regions and five loops; two loops are intracellular while the remaining three loops are extracellular. Therefore, the topology prediction of RmAQP2 is consistent with similar predictions previously done for other members of the AQP family (an in silico generated model of the predicted topology of the $R$. microplus AQP1 is shown in Fig. 1) $[1,2,4]$. Interestingly, this model also predicts the localization of the $\mathrm{N}$ - and C-terminal ends of RmAQP2 on the cytoplasmic side of the plasma membrane (Fig. 1), a characteristic feature of most known AQP proteins. Three-dimensional modeling [23] of the RmAQP2 protein suggests that it exists in the membrane as a tetramer.

\section{Differential pattern of transcription of the RmAQP2 gene among distinct tissues and live stages of the tick}

The transcription level of $R m A Q P 2$ was investigated by RT-qPCR in different tissues and stages of $R$. microplus fed on uninfected calves (B. bovis-free calves) (Fig. 2). In a previous study we tested several reference gene candidates from different tissues and stages of $R$. microplus, but none of them was found adequate for normalization of gene expression [14]. Therefore, in this study the expression level of $R m A Q P 2$ was normalized to the total amount of RNA used to generate the cDNA and the transcription level was calculated as a relative expression using the highest or lowest $C q$ value for $R m A Q P 2$ in a given sample as a control. Tick stages and tissues that were examined to evaluate gene expression included: unfed larvae (approximately 100 larvae per sample), engorged nymphs (10 nymphs per sample), unfed males (10 males per sample), and individual salivary glands, ovaries and guts of partially engorged females (at day 5 of feeding). We analyzed six biological replicates of each sample by RT-qPCR. Relative levels of expression of $R m A Q P 2$ were different in larvae $(2.5[ \pm 0.66])$, nymphs $(3.0[ \pm 0.23])$, female guts $(3.3[ \pm 0.99])$ and female salivary glands $(9.5[ \pm 1.73])$ (Fig. 2). However, no transcripts were detected in ovaries of partially engorged females and unfed male ticks. The relative gene expression of $R m A Q P 2$ in the female salivary glands samples was approximately 6 times higher $(P<0.001)$ than in larvae, nymphs and guts of females.

\section{Differential Expression of the RmAQP2 protein in different tick stages and tissues}

Following the detection of RmAQP2 mRNA in unfed larvae, engorged nymphs, and guts and salivary glands of partially engorged females, we investigated the presence of RmAQP2 protein in these tick stages and tissues (Fig. 3). Immunoblot assays were carried out using hybridoma supernatants. A hybridoma supernatant designated 147-677.13.11, used at a dilution 1:3, was selected for the immunoblot assay shown in Fig. 3. Anti-Bm86 polyclonal antibody was as a positive control used to demonstrate the presence of tick antigens in tick gut tissues for the immunoblot assays as previously described [27] (data not shown); however, we do not have a protein with associated antibody for protein expressed exclusively in the salivary gland as for aquaporin available for use as a salivary gland positive control. The aquaporin monoclonal antibody recognized proteins only in salivary glands of partially engorged females. In the blot shown in Fig. 3 shows that mAb 147-677.13.11 recognized $\mathrm{a} \approx 30 \mathrm{KDa}$ and an apparently more reactive $\approx$ $50 \mathrm{KDa}$ protein. The monomeric form of the RmAQP2 protein has a molecular mass predicted from the sequence of $\approx 30 \mathrm{KDa}$, likely corresponding to the smaller $\approx 30 \mathrm{KDa}$ band recognized by the $\mathrm{mAb}$ in the blot. Based on structural predictions we suggest (see discussion) that the larger $\approx 50 \mathrm{KDa}$ band may represent an $\mathrm{AQP}$ dimer. Interestingly, the antibody tested in the immunoblots didn't recognize any proteins in larvae, nymphs or guts of partially engorged females.

\section{Silencing of RmAQP2 in salivary glands and its effect on protein expression}

To assess RmAQP2 silencing in salivary gland, female ticks were injected with dsRNA. For the first experiment, adult female ticks were injected with one segment 
A

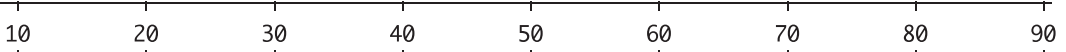

Rhipicephalus microplus AQP2 MKPNTVTRAWRQVTGCCIENTLARQALAEMVGTLVLTLVGDCVLASL----AVFQLGSVGLAAAPLGWGLAVFLGVLVAGGVSGAHLNP 85 Rhipicephalus microplus AQP1 Dermacentor variabilis AQP9 MKPNAVTRAWRRVGGCCIERPLARQALAEMVGTLVLTLVGDCVLASL-----AVFQLGSTGLAAGPLGWGLAVFLGVLVAGGVSGAHLNP 85 Rhipicephalus sanguineus AQP1-------MLDSVKIKNALVREVLAELLGTFVLVLFGNTVLAVV-----AFDRAPNLGYAAAFWGWALALTLGVLVAGGVSGAHLNP 74 Ixodes ricinus AQP1 Ixodes scapularis AQP1

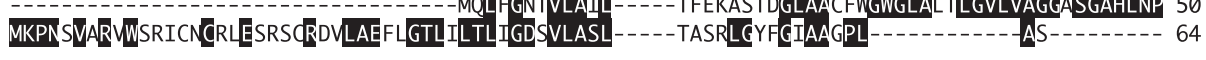

\begin{tabular}{|c|c|c|c|c|c|c|c|c|}
\hline 1 & $T$ & 1 & 1 & $T$ & $T$ & $T$ & 1 & $T$ \\
\hline 100 & 110 & 120 & 130 & 140 & 150 & 160 & 170 & 180 \\
\hline
\end{tabular}

Rhipicephalus microplus AQP2 AVTVALATIGKLGWCNVLAYVTAQYLGAFLASGLVYLVYADALSQVDVNLAIVYGTNATAPVFSCFPAPGVSTLTCLLDQTVSTAVLLLG 175 Rhipicephalus microplus AQP1 AVTLAQASVRKFPIAKVPLYFAAQYLGGFVGAALVFATYKDAIEHFDQGIRQVTGEKATAGIFATYPRPHVSTLTTFIDQVIATGIMMVC 159 Dermacentor variabilis AQP9 AVTVAMATVGKLAWCKVLAYVAAQYVGAFLASCLVYVVYTDALSQIDANLTTVYGINATAPVFSCFPAPGVSTLTCLVDQIVSTAVLLVG 175 Rhipicephalus sanguineus AQP1AVTVAVTTVGKFPURKIVPYMVAQYIGAFLASVILFITYRGALDNYDGGVRAVTGSNATAGIFASYPQEFVSTGNGLVDQIVGTALLVLA 164

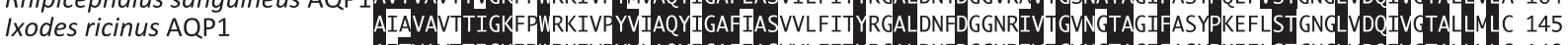
Ixodes scapularis AQP1 AITVAVTTIGKFPURKIVPYVLAQYIGAFIASVVLFITYRGALDNFDGGNRIVTGVNGTAGIFASYPKEFLSTGNGLVDQIVGTALLMLC 140 Ixodes scapularis AQP2

\begin{tabular}{lllllllll}
\cline { 2 - 4 } & 190 & 200 & 210 & 220 & 230 & 240 & 250 & 260
\end{tabular}

Rhipicephalus microplus AQP2 ICAITDGRNMA-VSRGQQPLLVGLTVSACMYAFSYNCGNPLNPARDLAPRIFTAMCGWGSAVFSFRSYNWFWVPVVGPHLGAVIGVWIYK 264

Rhipicephalus microplus AQP1 VEAIGDTRNFGGIPPHIHPICLGLMIMAIIFSFAYNCMCPLNPARDISPRLFTLMAGWCPETFTLRGWNYWWVLLGPHIGAILGVWLY 249 Dermacentor variabilis AQP9 ICAITDTRNMD-VSRGQQPLLVGLTVSACMYAFSYNCGNPLNPARDLAPRIFTAMSGWGSAVFSFRAYNWFWVPVVGPHLGAVIGVWIYK 264 Rhipicephalus sanguineus AQP1 ILAITDSRNMA-VPQGVPLFIGLALGALILCFGYNCCAPLNPARDLGPRVFTAMAGWGVEVFSYRDYNWFWVPIVGPHIGAIVGAWLYT 253 Ixodes ricinus AQP1 Ixodes scapularis AQP1 Ixodes scapularis AQP2

ILAITDARNMA-VPQGVQPLFI FALAAIILSFGYNCGAPLNPARDLAPRVFTAMAGWGGEVFSYRDYNWFWVPILGPH IGAILGAWIYT 234
ILAITDARNMA-VPQGVQPLFIGFALAAIILSFGYNCGAPLNPARDLAPRVFTAMAGWGGEVFSFRDYNWFWVPILGPHMGAILGAWIYT 229
ICAIVDPKNMA-VLKGHQPLLICFAVAACMYAFSYNCGNPLNPARDLAPRIFTAMAGWCCDVFSIRGYNWFWVPVVGPHVGGVVGVIYK 235

\begin{tabular}{rlllllll}
\cline { 2 - 5 } & 280 & 290 & 300 & 310 & 320 & 330 & 340 \\
\hline
\end{tabular}

Rhipicephalus microplus AQP2 LAV Rhipicephalus microplus AQP1
Dermacentor variabilis AQP9 Rhipicephalus sanguineus AQP1 Ixodes ricinus AQP1 Ixodes scapularis AQP1 Ixodes scapularis AQP2
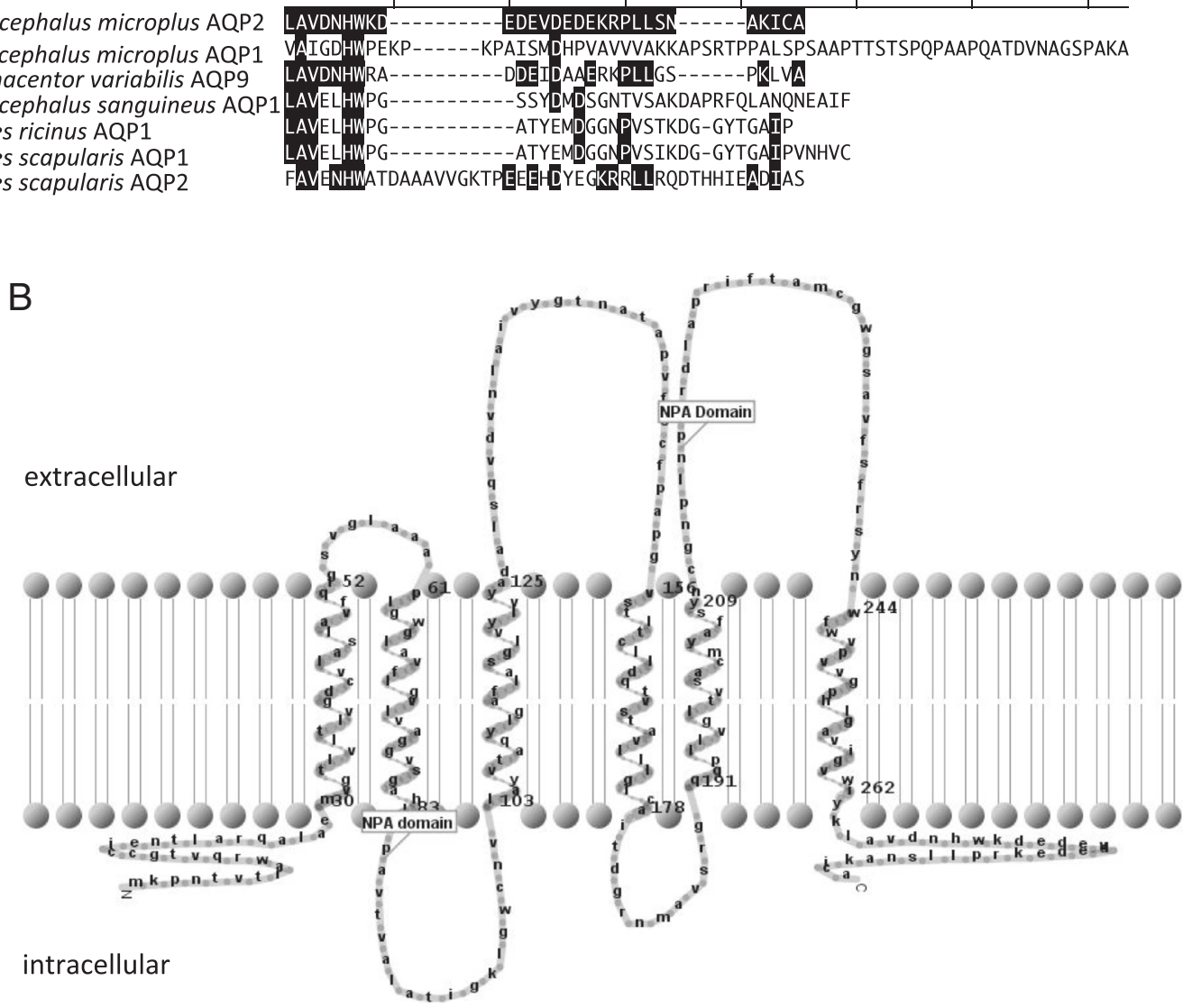

Fig. 1 (See legend on next page.) 
(See figure on previous page.)

Fig. 1 Multiple sequence alignments and topology prediction of RmAQP2. Panel a shows the amino acid alignment of RmAQP2 [GenBank ID: KP406519], R. microplus AQP1 [GenBank ID: KJ626366.1], Dermacentor variabilis AQP9 [GenBank ID: ABI53034.1], Rhipicephalus sanguineus AQP1 [GenBank ID: CAR66115.1], Ixodes ricinus AQP1 [GenBank ID: CAX48964.1], Ixodes scapularis AQP1 [GenBank ID: XP_002399532.1] and Ixodes scapularis AQP2 [GenBank ID: XP_002400655.1]. Black boxes in Panel A indicate identical amino acid residues when comparing RmAQP2 and the other sequences. Panel b shows the topology prediction of RmAQP2 indicating the presence of 6 transmembrane-spanning regions, 3 extracellular loops, 2 intracellular loops and 2 NPA AQP signature domains

of dsRNA from the $3^{\prime}$ end of the gene and fed on $B$. bovis-infected calf. For the second experiment, adult female ticks were injected with two segments of dsRNA, one from the $5^{\prime}$ end and one from the $3^{\prime}$ end of the gene, and then fed to repletion on an uninfected calf $(B$. bovis-free animal). Both dsRNA fragments were approximately $400 \mathrm{bp}$ in length and their respective location on the RmAQP2 gene is represented in Additional file 1: Figure S1. To assess silencing, salivary glands were examined from six individual ticks (representing 6 biological replicates) by RT-qPCR after 5 days of feeding on cattle. Similar levels of $R m A Q P 2$ silencing were obtained when either one or two dsRNA fragments were used for silencing. RmAQP2 was silenced $92.8 \%( \pm 0.26 \%)$ and $99.3 \%( \pm 3.70 \%)$ in ticks injected with either one dsRNA or the two non-overlapping dsRNA fragments, respectively (Fig. 4). Next, we investigate the effect of $R m A Q P 2$ silencing on protein expression. Female ticks injected with two dsRNA fragments were fed on a calf for 5 days, dissected and individual salivary glands collected. Figure 5 demonstrates the absence of RmAQP2 protein in salivary glands of dsRNA-injected ticks. As expected,

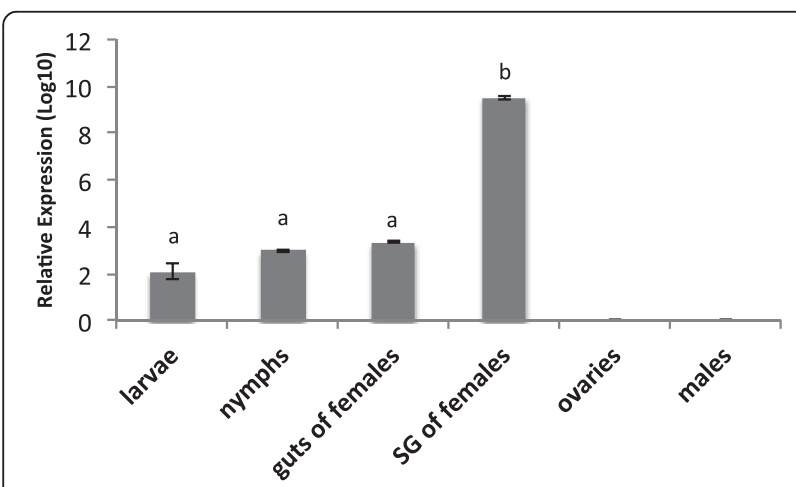

Fig. 2 Expression of the RmAQP2 gene in unfed larvae, engorged nymphs, unfed males, and salivary glands, gut and ovaries of partially engorged (day 5 of feeding) $R$. microplus females. Six biological replicates of unfed larvae (approximately 100 larvae per sample), engorged nymphs (approximately 10 nymphs per sample), unfed males (approximately 10 males per sample), and individual salivary glands, ovaries and guts of partially engorged females (at day 5 of feeding) were analyzed by reverse transcriptase quantitative real-time PCR. The transcription level of RmAQP2 was calculated as relative quantity using the delta $C_{t}$ comparative method normalized by the total amount of RNA used to generate the cDNA. Different letters $(a, b)$ above the bars indicate significant statistical differences (one-way ANOVA, Tukey post hoc test, $P<0.001$ ) the presence of RmAQP2 protein was demonstrated in salivary glands of the control non-silenced ticks (Fig. 5). Collectively, the data demonstrated gene silencing and abrogation of protein expression as a consequence of injecting ticks with dsRNA identical to $R m A Q P 2$.

\section{Effect of RmAQP2 silencing on tick fitness}

After removal of the ticks for assessment of silencing and protein expression, the reminder of the ticks in the two independent experiments were allowed to feed to repletion to investigate the in vivo biological effect of RmAQP2 silencing. For the first experiment, adult female ticks were injected with one segment of dsRNA (from the 3' end of the gene) (Additional file 1: Figure S1) and fed on B. bovis-infected calf. For the second experiment, adult female ticks were injected with two segments of dsRNA (from both the $5^{\prime}$ and $3^{\prime}$ ends of the gene) (Additional file 1: Figure S1) and fed to repletion on an uninfected calf (B. bovis-free animal). In both experiments after the dsRNA-injected females were fed to repletion, the percentage of engorged females, weight of engorged females, oviposition rate, egg masses, percentage of hatching and percentage of larvae survival were evaluated (Tables 2 and 3). Considering the ticks that fed on a calf during acute $B$. bovis infection (experiment 1 ), identical numbers of females from both, the $R m A Q P 2$ silenced group and control group, fed to repletion. The average weight of the $R m A Q P 2$-silenced engorged females was $361.8 \mathrm{mg}$ and significantly higher $(P<0.05)$ than the control group (342.4 mg). Silencing of $R m A Q P 2$ had no effect on the percentage of oviposition and egg masses between the two groups of ticks. In contrast, only $50 \%$ of the egg masses hatched compared to $90.2 \%$ hatching of the control group. Strikingly, none of the larvae survived in the $R m A Q P 2$-silenced group compared to $100 \%$ larvae survival in the control group (Table 2). Considering the ticks that fed on the uninfected calf (experiment 2), identical numbers of females from both, the RmAQP2 silenced group and control group, fed to repletion. The average weight of engorged females in the RmAQP2 silenced group was $323.3 \mathrm{mg}$ and significantly higher $(P<0.05)$ than the average weight of engorged females in the control group (305.6 mg). Oviposition rate was not affected by RmAQP2 silencing; however, egg mass weight was significantly lower $(P<0.05)$ in the silenced group $(134.7 \mathrm{mg})$ than in 


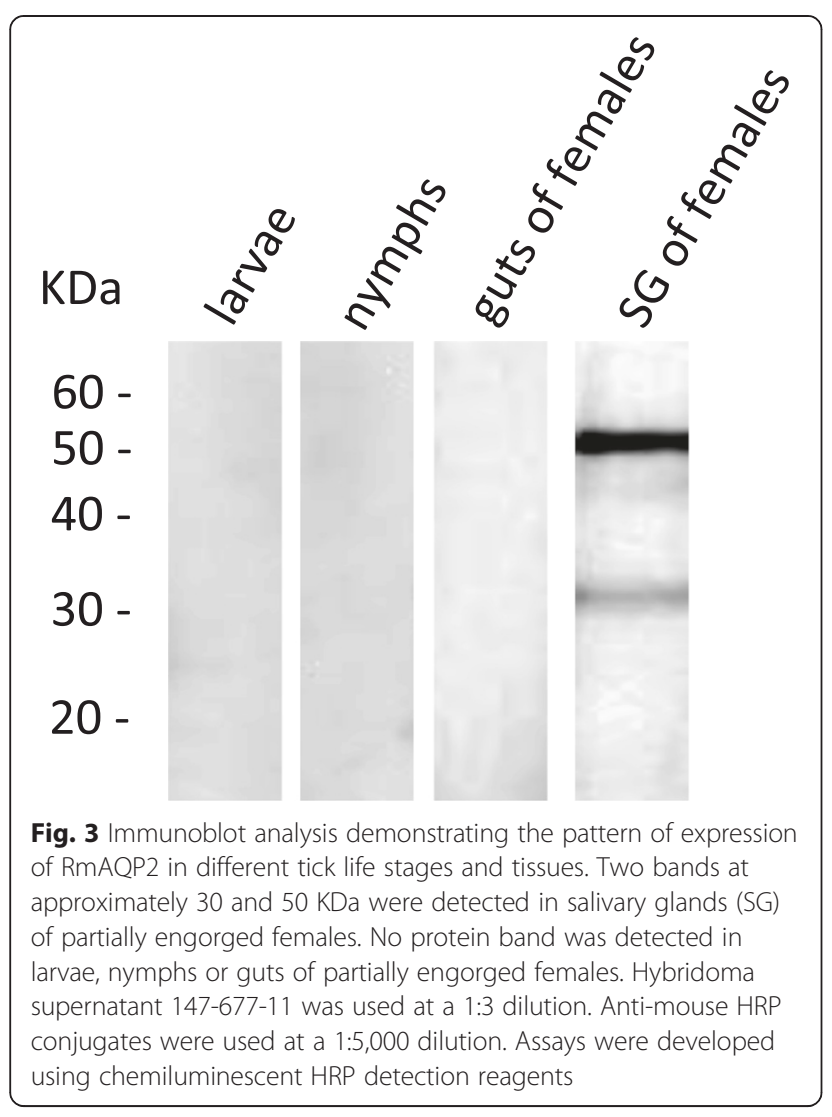

the control group (203.4 mg). Interestingly, the percentage of hatching was significantly reduced $(P<0.05)$ in the silenced group $(81.2 \%)$ compared to the control group (97.7\%); however, $100 \%$ of the larvae survived in both the silenced and control groups (Table 3).

\section{Discussion}

In the present study we investigated the pattern of expression of a newly identified aquaporin 2 of $R$. microplus ticks. RmAQP2 transcript was demonstrated in unfed larvae, engorged nymphs, and salivary glands and guts of partially engorged female ticks. Interestingly, however, among the ticks tissues and stages investigated, RmAQP2 protein was found only differentially expressed in the salivary glands of partially engorged females. In vivo functional analyses showed that silencing of $R m A Q P 2$ decreased tick fitness and this effect was more pronounced in ticks fed on a calf during acute $B$. bovis infection. Noteworthy, none of the larval progeny from the RmAQP2-silenced females that were fed on a B. bovisinfected calf survived. Considering that $R$. microplus larvae transmit $B$. bovis, these data may have implications for the control of tick-borne parasites [6].

Since $R$. microplus is a one-host tick that infests the host as larvae and remains until the adult females have fed to repletion, we did not think it was meaningful to assess all tick life stages of the tick for aquaporin expression. The adult female takes the majority of the blood. It is that stage that is primarily responsible for acquisition of $B$. bovis and it is also that stage that causes the most direct damage to the host by feeding. B. bovis is passed from the adult female transovarially to the larval offspring and it is the larval stage that transmits B. bovis. Consequently, the most biologically relevant stages of the tick for acquisition and transmission of $B$. bovis are the adult female and the larvae, respectively. Adult females are the most biologically relevant stages with regard to damage cause by tick feeding. Therefore, our investigations focused primarily on these two stages for expression of the aquaporin gene.

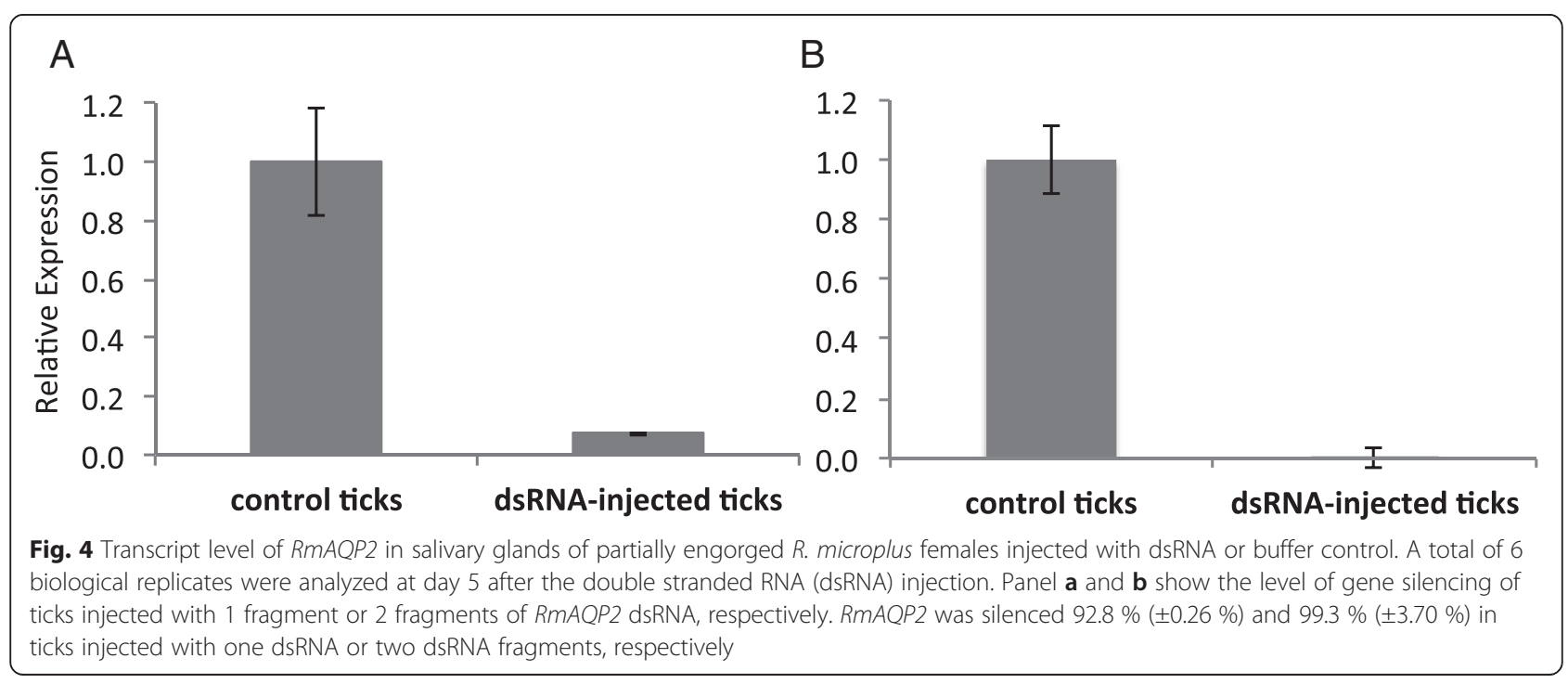




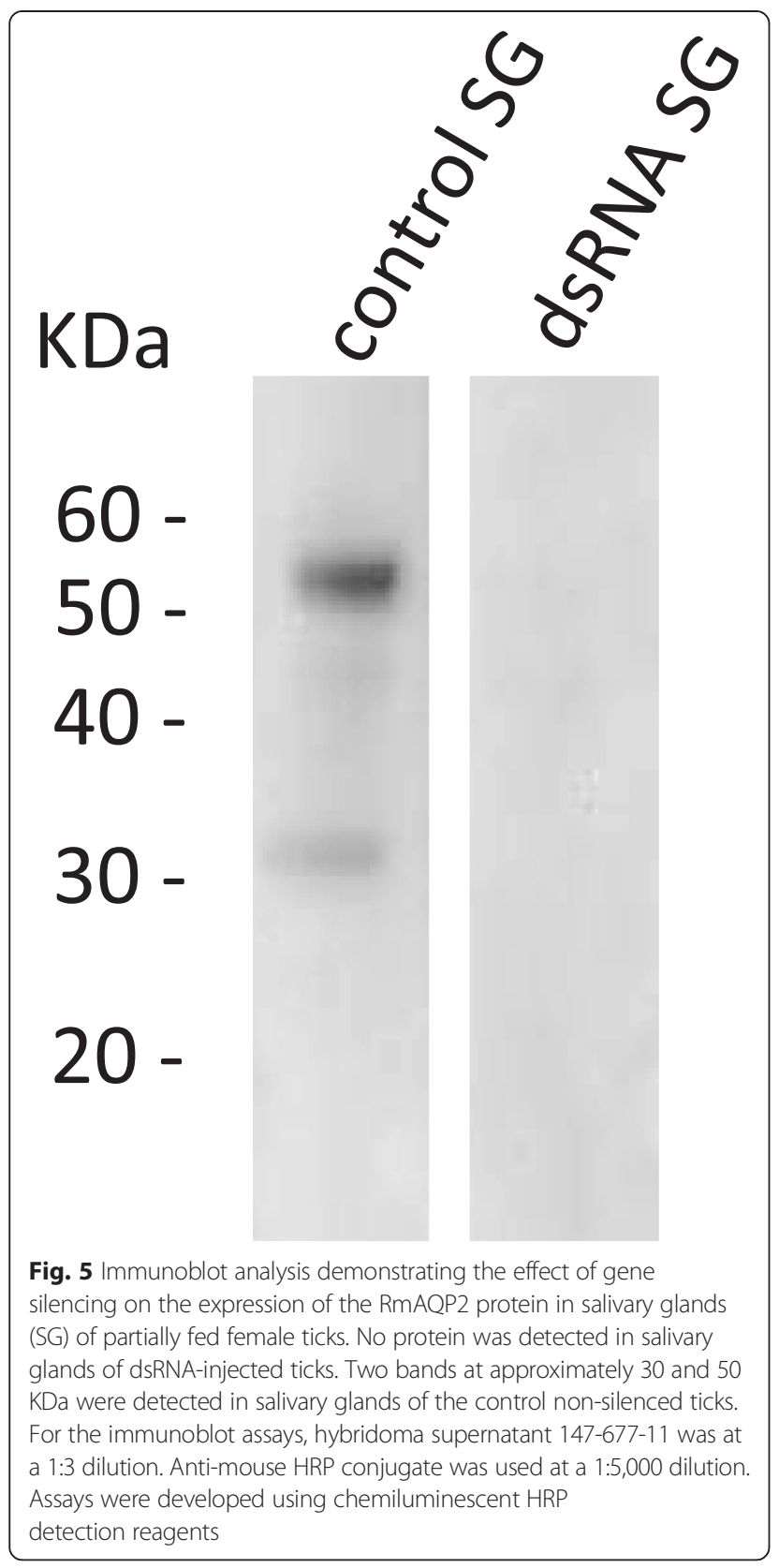

Table 2 Biological effect of RmAQP2 silencing on tick fed on a calf during acute B. bovis infection (experiment 1)

\begin{tabular}{lllllll}
\hline & $\begin{array}{l}\text { Percentage of } \\
\text { engorged females }\end{array}$ & $\begin{array}{l}\text { Weight }(\mathrm{mg}) \text { of } \\
\text { engorged females }\end{array}$ & Oviposition rate & Egg mass (mg) & $\begin{array}{l}\text { Percentage } \\
\text { of hatching }\end{array}$ & $\begin{array}{c}\text { Percentage of } \\
\text { larvae survival }\end{array}$ \\
\hline $\begin{array}{l}\text { Control females fed on } \\
\text { a B. bovis-infected calf }\end{array}$ & $46.1 \%(83 / 180)$ & $342.4( \pm 5.6)$ & $98.7 \%(82 / 83)$ & $141.1( \pm 42.3)$ & $90.2 \%$ & $100 \%(74 / 74)$ \\
$\begin{array}{l}\text { Silenced females fed on } \\
\text { a B. bovis-infected calf }\end{array}$ & $46.1 \%(83 / 180)$ & $361.8( \pm 7.0)^{\mathrm{a}}$ & $96.3 \%(80 / 83)$ & $139.5( \pm 50.5)$ & $50.0 \%{ }^{\mathrm{b}}$ & 0 \\
\hline
\end{tabular}

Members of the AQP family have recently been identified in several arthropods including tick species, such as $D$. variabilis, $R$. sanguineus, I. ricinus, I. scapularis and $I$. scapularis $[28,29]$. Here we described a newly identified AQP2 of $R$. microplus ticks. The presence of two NPA domains and the level of amino acid identity between RmAQP2 and other AQPs prompt us to argue about a potential evolutionary conserved function for these proteins. The remarkable similarity of topology among AQP members in different species, including RmAQP2 described in this study, also supports the prediction of a putative conserved function $[1,2,4]$. A cysteine located five residues upstream of the second NPA motif suggests that RmAQP2 is likely to be sensitive to mercury as demonstrated for other AQP members [1]. This may set the rationale for the design of novel acaricides targeting AQP to control $R$. microplus infestation and $R$. microplus-borne parasites. It has been proposed that AQPs play a critical osmoregulatory function in blood-feeding arthropods during saliva production, intake and excretion of water during blood digestion, reproduction and egg development, and off-host environment stress tolerance [3]. Salivary glands are of vital importance for tick biology, acting as osmoregulatory organs for both off-host and on-host stages of their life cycle [29]. We postulate that AQPs expressed uniquely in the tick salivary glands may play an important role in the osmoregulatory stress during blood feeding and digestion. RmAQP2-silenced female ticks had a significant increase in body weight at the time of repletion compared to control nonsilenced ticks, suggesting a level of involvement of RmAQP2 in osmoregulatory mechanisms. Additionally, the increased body weight implies that ticks accumulate more liquids in the absence of RmAQP2. Despite being heavier, the RmAQP2-silenced group produced egg masses of reduced weight and reduced hatching success when compared with the control group, suggesting a negative correlation between intake of blood and generation of viable larval offspring.

Considering that RmAQP2 protein was only found in salivary glands, it is reasonable to assume that this AQP plays an essential role in osmoregulation during blood feeding and digestion. The data showed that knockdown of $R m \mathrm{AQP} 2$ interfered with the osmoregulatoty system of $R$. microplus during feeding as demonstrated by the

${ }^{\mathrm{b}}$ Chi-squared test $(P<0.05)$

${ }^{a} t$ test $(P<0.05)$ 
Table 3 Biological effect of RmAQP2 silencing on tick fed on an uninfected calf (experiment 2)

\begin{tabular}{lllllll}
\hline & $\begin{array}{l}\text { Percentage of } \\
\text { engorged females }\end{array}$ & $\begin{array}{l}\text { Weight }(\mathrm{mg}) \text { of } \\
\text { engorged females }\end{array}$ & Oviposition rate & Egg mass (mg) & $\begin{array}{l}\text { Percentage } \\
\text { of hatching }\end{array}$ & $\begin{array}{c}\text { Percentage of } \\
\text { larvae survival }\end{array}$ \\
\hline $\begin{array}{l}\text { Control females fed on } \\
\text { an uninfected calf }\end{array}$ & $37.7 \%(68 / 180)$ & $305.6( \pm 6.1)$ & $98.5 \%(67 / 68)$ & $203.4( \pm 39.0)$ & $97.7 \%$ & $100 \%$ \\
$\begin{array}{l}\text { Silenced females fed on } \\
\text { an uninfected calf }\end{array}$ & $51.1 \%(92 / 180)$ & $323.3^{\mathrm{a}}( \pm 5.7)$ & $94.5 \%(87 / 92)$ & $134.7^{\mathrm{a}}( \pm 63.6)$ & $81.2 \%{ }^{\mathrm{b}}$ & $100 \%$ \\
\hline
\end{tabular}

${ }^{a} t$ test $(P<0.05)$

${ }^{\mathrm{b}}$ Chi-squared test $(P<0.05)$

increased body weight in the gene-silenced engorged females. Effect on tick fitness was even more pronounced in ticks fed on a B. bovis-infected calf perhaps due to the deleterious presence of the apicomplexa parasite infection [6]. Additionally, it is reasonable to speculate about a potential cumulative effect of $R m A Q P 2$ silencing throughout the tick life cycle considering that none of the larval progeny survived from the silenced females that fed on the calf during acute B. bovis infection. Here we also show that dsRNA injection abolished the expression of RmAQP2 protein completely in salivary gland of partially engorged females at 5 days of feeding. This result suggests a possible rapid turnover of RmAQP2 in salivary glands. Rapid turnover of AQPs has been previously demonstrated both in animal and plant tissues and shown to be more pronounced during infections or when organisms are under stress [30-32]. The extreme osmoregulatory changes in salivary glands of ticks during feeding could induce a high level of physiological turnover of AQPs, as demonstrated by the present data for RmAQP2. It was recently demonstrated that $A Q P 1$ mRNA of $I$. ricinus ticks is expressed only in female tissues involved in water flux, such as guts, rectal sac and especially abundant in salivary glands [29]. Here we demonstrated a similar pattern of expression for RmAQP2 transcripts. However, RmAQP2 protein was found in salivary glands. Therefore, it is plausible to assume the presence of a mechanism to suppress translation of $R m A Q P 2$ in female guts, larvae and nymphs. Silencing of I. ricinus $A Q P 1$ affected feeding performance, but the ticks remained feeding on the host with subsequent potential for pathogen transmission. Therefore, the authors concluded that I. ricinus $A Q P 1$ is not a suitable target to control tick infestation or block transmission of tick-borne diseases [29]. Our data showed that RmAQP2-silenced female ticks ingested more blood and consequently showed increased engorgement body weight. Hatching and larvae survival were also affected by gene silencing and most importantly, none of the larval progeny from the RmAQP2silenced females fed on a $B$. bovis-infected calf survived. Differences in site and level of expression, and permeability may explain the discrepancies between the present study and the previous published data regarding I. ricinus $A Q P 1$ [29]. Another recent study evaluated AQP1 of $R$. microplus as an anti-tick vaccine [16]. The authors demonstrated that vaccination of cattle with recombinant $R$. microplus AQP1 effectively reduced the number of adult female ticks that fed to repletion.

Based on the amino acid sequence, RmAQP2 has a predicted molecular weight of $\approx 30 \mathrm{KDa}$. However, preliminary in silico analysis using NetOGlyc [33] and NetPhos [34] revealed the presence of several putative glycosylation and phosphorylation sites on RmAQP2 (data not shown). Post-translational modifications of AQP have been previously described [35-37] and it may be one explanation for the detection of larger protein products in the immunoblot assays. Structural predictions suggest that this protein may exist in the membrane as a tetramer and the multiple banding pattern in the blot could represent varying stages of the breakdown of this tetrameric structure; cleavage of the intracellular tails of the RmAQP2 would result in a molecular mass of $\approx 25 \mathrm{KDa}$, suggesting that the larger $50 \mathrm{KDa}$ band could be a dimer of two molecules. In additional blots (not shown) we also see another band above $100 \mathrm{KDa}$, which could represent the tetramer. Another possibility to explain the difference between expected and observed molecular weight is that the $R m A Q P 2$ cDNA sequence obtained from the $R$. microplus gene index project may be incomplete at the $5^{\prime}$ end. This sequence was derived from an EST project and although it has two stop codons in the 3 ' end of the sequence, a stop codon inframe with the presumed start ATG codon is not present. Nevertheless, this possibility is unlikely considering that the topology analyses revealed a typical predicted conformation for the AQP family [3, 4, 28]. Regardless of these considerations, the key finding is that all of the bands recognized by the monoclonal antibody raised to the RmAQP2 peptides are absent in the silenced ticks, suggesting that silencing has completely abrogated protein expression. It was beyond the scope of this study to determine the type of cell(s) in the tick salivary glands expressing the RmAQP2 protein; however, the immunoblot data clearly demonstrate that salivary glands are the unique site of expression of this AQP in R. microplus.

RNA interference has been widely used to investigate gene function in arthropods. However, off-target effects have been described in some species [38] and cannot be entirely ruled out as the cause of the results observed in 
this experiment. The $R$. microplus genome sequence is not available and the alignment analyses are restricted to sequences listed in databases. Blast analysis of the dsRNA sequences used in this study did not reveal significant homology to any known tick sequence other than $R m A Q P 2$. Additionally, gene silencing was validated by RT-qPCR and RmAQP2 was silenced at similar levels when we used either one or two different segments of dsRNA identical to the target gene. Taken together, these aspects support the data and represent the best possible strategy to make solid scientific observations regarding the biological effect of RmAQP2 silencing in R. microplus.

\section{Conclusion}

Here we demonstrate the pattern of expression of a newly identified aquaporin 2 gene of $R$. microplus ticks. RmAQP2 transcripts were present in unfed larvae, engorged nymphs, and salivary glands and guts of partially engorged female ticks. However, among the distinct adult tissues and life stages tested, RmAQP2 protein was only found in salivary glands of partially engorged females. In vivo investigation demonstrated that silencing of $R m A Q P 2$ decreased tick fitness. The effect of RmAQP2 silencing on tick fitness was even more pronounced in females fed on a B. bovis-infected calf and none of their larval progeny survived. Therefore, we conclude that RmAQP2 is involved in osmoregulation during feeding and is a potential target for the development of novel strategies for the control of $R$. microplus and R. microplus-borne diseases.

\section{Additional file}

Additional file 1: Figure S1. Sequence of RmAQP2 CDNA [GenBank ID: KP406519]. Blue and green lines indicate the $5^{\prime}$ double stranded RNA (dsRNA) (Blue) and 3' dsRNA segments (green), respectively. Red lines indicate the regions utilized to design synthetic peptides for monoclonal antibody production. (PDF $66 \mathrm{~kb}$ )

\section{Competing interests}

The authors declare that they have no competing interests.

\section{Authors' contribution}

$\mathrm{HEH}$ and RGB designed and performed the experiment, and wrote the first draft of the manuscript. MWU, CES and GAS designed and performed the experimental procedures involving ticks. FDG identified the target gene used in this study and contributed to the experimental design. FKA designed and supervised the experiment. All authors made intellectual contribution regarding data interpretation, and reviewed and approved the final version of the manuscript.

\section{Acknowledgments}

We thank Kathy Mason, Ralph Horn and James Allison for excellent technical assistance. This work was supported by USDA-ARS-CRIS project number 5348-32000-034-00D. H. E. Hussein was financially supported by the Egyptian Ministry of High Education.

\section{Author details}

'Department of Veterinary Microbiology and Pathology, Washington State University, Pullman, WA 99164, USA. ${ }^{2}$ Department of Entomology, Faculty of
Science, Cairo University, Giza 12613, Egypt. ${ }^{3}$ Animal Disease Research Unit, USDA-ARS, Washington State University, 3003 ADBF, P.O. Box 646630, Pullman, WA 99164, USA. ${ }^{4}$ USDA-ARS, Knipling Bushland US Livestock Insect Research laboratory, 2700 Fredericksburg Road, Kerrville, TX 78028, USA.

${ }^{5}$ USDA-ARS Veterinary Pest Genomics Center, Kerrville, TX, USA. ${ }^{6}$ School of Molecular Biosciences, Washington State University, Pullman, WA 99164, USA.

Received: 14 July 2015 Accepted: 24 November 2015

Published online: 02 December 2015

\section{References}

1. Borgnia M, Nielsen S, Engel A, Agre P. Cellular and molecular biology of the aquaporin water channels. Annu Rev Biochem. 1999;68:425-58.

2. Gonen T, Walz T. The structure of aquaporins. Q Rev Biophys. 2006;39:361-96.

3. Benoit JB, Hansen IA, Szuter EM, Drake LL, Burnett DL, Attardo GM. Emerging roles of aquaporins in relation to the physiology of blood-feeding arthropods. J Comp Physiol B. 2014;184(7):811-25.

4. Campbell EM, Ball A, Hoppler S. Invertebrate aquaporins: a review. J Comp Physiol B. 2008;178:935-55.

5. Kaufman WR, Phillips JE. Ion and water balance in the ixodid tick Dermacentor andersoni. I. routes of ion and water excretion. J Exp Biol. 1973;58:523-36.

6. Friedhoff KT. Transmission of babesia. In: Ristic M, editor. Babesiosis of Domestic Animals and Man. Boca Raton, FL, USA: CRC Press; 1988. p. 23-52.

7. Mahoney DF, Mirre GB. A note on the transmission of Babesia bovis (syn B Argentina) by the one-host tick, Boophilus microplus. Res Vet Sci. 1979;26:253-4.

8. Bock R, Jackson L, de Vos A, Jorgensen W. Babesiosis of cattle. Parasitology. 2004;129(Suppl):S247-69.

9. Fragoso H, Rad PH, Ortiz M, Rodriguez M, Redondo M, Herrera L, et al. Protection against Boophilus annulatus infestations in cattle vaccinated with the B. microplus Bm86-containing vaccine Gavac. Vaccine. 1998;16:1990-2.

10. Jonsson NN, Matschoss AL, Pepper P, Green PE, Albrecht MS, Hungerford JJ, et al. Evaluation of TickGARDPLUS, a novel vaccine against Boophilus microplus, in lactating Holstein- Friesian cows. Vet Parasitol. 2000;88:275-85.

11. Miller RJ, Davey RB, George JE. First report of organophosphateresistant Boophilus microplus (Acari: Ixodidae) within the United States. J Med Entomol. 2005;42:912-7.

12. de la Fuente J, Almazán C, Canales M, de la Lastra JMP, Kocan KM, Willadsen P. A ten-year review of commercial vaccine performance for control of tick infestations on cattle. Anim Health Res Rev. 2007;8:23-8.

13. Bastos RG, Ueti MW, Guerrero FD, Knowles DP, Scoles GA. Silencing of a putative immunophilin gene in the cattle tick Rhipicephalus (Boophilus) microplus increases the infection rate of Babesia bovis in larval progeny. Parasites Vectors. 2009;2:57

14. Goff WL, Molloy JB, Johnson WC, Suarez CE, Pino I, Rhalem A, et al. Validation of a competitive enzyme-linked immunosorbent assay for detection of antibodies against Babesia bovis. Clin Vaccine Immunol. 2006;13:1212-6.

15. Stiller D, Goff WL, Johnson LW, Knowles DP. Dermacentor variabilis and Boophilus microplus (Acari: Ixodidae): experimental vectors of Babesia equi to equids. J Med Entomol. 2002;39:667-70.

16. Guerrero FD, Andreotti R, Bandele KG, Cunha RC, Miller RJ, Yeater K, et al. Rhipicephalus (Boophilus) microplus aquaporin as an effective vaccine antigen to protect against cattle tick infestations. Parasites Vectors. 2014;7:475.

17. Guerrero FD, Miller RJ, Rousseau ME, Sunkara S, Quackenbush J, Lee Y, et al. BmiGl: A database of CDNAs expressed in Boophilus microplus, the tropical/ southern cattle tick. Insect Biochem Mol Biol. 2005;35:585-95.

18. McCooke JK, Guerrero FD, Barrero RA, Black M, Hunter A, Bell C, et al. The mitochondrial genome of a Texas outbreak strain of the cattle tick, Rhipicephalus (Boophilus) microplus, derived from whole genome sequencing Pacific Biosciences and Illumina reads. Gene. 2015;571:135-41.

19. Goff WL, Johnson WC, Cluff CW. Babesia bovis immunity. In vitro and in vivo evidence for IL-10 regulation of IFN-gamma and iNOS. Ann N Y Acad Sci. 1998;849:161-80.

20. Letunic I, Doerks T, Bork P. SMART: recent updates, new developments and status in 2015. Nucleic Acids Res. 2015;43:Database issue D257-D260.

21. Chaturvedi N, Shanker S, Singh VK, Sinha D. Hidden markov model for the prediction of transmembrane proteins using MATLAB. Bioinformation. 2011; 7:418-21.

22. Spyropoulos IC, Liakopoulos TD, Bagos PG, Hamodrakas SJ. TMRPres2D: high quality visual representation of transmembrane protein models. Bioinformatics. 2004;20:3258-60. 
23. Biasini M, Bienert S, Waterhouse A, Arnold K, Studer G, Schmidt T, et al. SWISS-MODEL: modelling protein tertiary and quaternary structure using evolutionary information. Nucleic Acids Res. 2014;42(W1):W252-8.

24. Bustin SA, Benes V, Garson JA, Hellemans J, Huggett J, Kubista M, et al. The MIQE guidelines: minimum information for publication of quantitative real-time PCR experiments. Clin Chem. 2009:55(4):611-22.

25. Bastos RG, Suarez CE, Laughery JM, Johnson WC, Ueti MW, Knowles DP. Differential expression if three members of the multidomain CCp family in Babesia bigemina. Babesia bovis and Theileria equi. PLoS ONE. 2013;8:e67765.

26. Yokoyama WM. Production of monoclonal antibodies. In: Coligan JE, editor. Current protocols in immunology. 1st ed. New York: Wiley Intersciences Inc; 1994. p. 2.5.1-2.5.17

27. Laughery JM, Knowles DP, Schneider DA, Bastos RG, McElwain TF, Suarez CE. Targeted Surface Expression of an Exogenous Antigen in Stably Transfected Babesia bovis. PLoS ONE. 2014;9:e97890.

28. Ball A, Campbell EM, Jacob J, Hoppler S, Bowmana AS. Identification, functional characterization and expression patterns of a water-specific aquaporin in the brown dog tick, Rhipicephalus sanguineus. Insect Biochem Mol Biol. 2009;39:105-12.

29. Campbell EM, Burdin M, Hoppler S, Bowmana AS. Role of an aquaporin in the sheep tick Ixodes ricinus: Assessment as a potential control target. Int J Parasitol. 2010:40:15-23.

30. Fleurat-Lessard P, Michonneau P, Maeshima M, Drevon JJ, Serral R. The distribution of aquaporin subtypes (PIP1, PIP2 and g-TIP) is tissue dependent in soybean (Glycine max) root nodules. Ann Bot. 2005;96:457-60.

31. Castañeyra-Ruiz L, González-Marrero I, González-Toledo JM, Castañeyra-Ruiz A, Paz-Carmona H, Castañeyra-Perdomo A, et al. Aquaporin-4 expression in the cerebrospinal fluid in congenital human hydrocephalus. Fluids Barriers CNS. 2013;10:18.

32. De Bellis M, Pisani F, Mola MG, Basco D, Catalano F, Nicchia GP, et al. A novel human aquaporin-4 splice variant exhibits a dominant-negative activity: a new mechanism to regulate water permeability. Mol Biol Cell. 2014; $25: 470-80$

33. Steentoft C, Vakhrushev SY, Joshi HJ, Kong Y, Vester-Christensen MB, Schjoldager $\mathrm{KT}$, et al. Precision mapping of the human O-GalNAc glycoproteome through SimpleCell technology. EMBO J. 2013;32(10):1478-88.

34. Blom N, Gammeltoft S, Brunak S. Sequence- and structure-based prediction of eukaryotic protein phosphorylation sites. J Mol Biol. 1999;294(5):1351-62.

35. Ball LE, Garland DL, Crouch RK, Schey KL. Post-translational modifications of aquaporin 0 (AQP0) in the normal human lens: Spatial and temporal occurrence. Biochemistry. 2004;43:9856-65.

36. Moeller HB, Olesen ETB, Fenton RA. Regulation of the water channel aquaporin-2 by posttranslational modification. Am J Physiol Renal Physiol. 2011;300:1062-73.

37. Tamma G, Ranieri M, Di Mise A, Centrone M, Svelto M, Valenti G. Glutathionylation of the aquaporin-2 water channel: a novel posttranslational modification modulated by the oxidative stress. J Biol Chem. 2014;289:27807-13.

38. Ma Y, Creanga A, Lum L, Beachy PA. Prevalence of off-target effects in Drosophila RNA interference screens. Nature. 2006:443:359-63.

\section{Submit your next manuscript to BioMed Central and we will help you at every step:}

- We accept pre-submission inquiries

- Our selector tool helps you to find the most relevant journal

- We provide round the clock customer support

- Convenient online submission

- Thorough peer review

- Inclusion in PubMed and all major indexing services

- Maximum visibility for your research

Submit your manuscript at www biomedcentral com/submit 